\title{
Development and Validation of an Ion-Pair HPLC-UV Method for the Quantitation of Quinoline and Indoloquinoline Alkaloids in Herbal and Pharmaceutical Antimalarial Formulations
}

\author{
Samuel Oppong Bekoe $\mathbb{D}^{1},{ }^{1}$ Emmanuel Orman $\mathbb{D},{ }^{2}$ Samuel Awenteka Adjabui, ${ }^{3}$ \\ Abena Amponsaa Brobbey, ${ }^{1}$ James Oppong-Kyekyeku, ${ }^{1}$ \\ Kwabena Frimpong-Manso Opuni $\mathbb{D}^{4},{ }^{4}$ Noble Kuntworbe, ${ }^{5}$ and Samuel Asare-Nkansah ${ }^{1}$ \\ ${ }^{1}$ Department of Pharmaceutical Chemistry, Faculty of Pharmacy and Pharmaceutical Sciences, \\ Kwame Nkrumah University of Science and Technology, Kumasi, Ghana \\ ${ }^{2}$ Department of Pharmaceutical Chemistry, School of Pharmacy, University of Health and Allied Sciences, Ho, Ghana \\ ${ }^{3}$ Department of Quality Assurance, Ernest Chemists Limited, Accra, Ghana \\ ${ }^{4}$ Department of Pharmaceutical Chemistry, School of Pharmacy, University of Ghana, Legon, Ghana \\ ${ }^{5}$ Department of Pharmaceutics, Faculty of Pharmacy and Pharmaceutical Sciences, \\ Kwame Nkrumah University of Science and Technology, Kumasi, Ghana
}

Correspondence should be addressed to Samuel Oppong Bekoe; sobek03@gmail.com and Emmanuel Orman; eorman@uhas.edu.gh

Received 30 October 2021; Revised 19 January 2022; Accepted 28 January 2022; Published 2 March 2022

Academic Editor: Shayessteh Dadfarnia

Copyright (C) 2022 Samuel Oppong Bekoe et al. This is an open access article distributed under the Creative Commons Attribution License, which permits unrestricted use, distribution, and reproduction in any medium, provided the original work is properly cited.

Quinine- and cryptolepine-based antimalarials serve as valuable alternatives to artemisinin-based combination therapies (ACTs) in Ghana. Their use, however, is associated with adulteration and substandard quality challenges. An HPLC method targeting quinoline and indoloquinoline antimalarial alkaloids was developed, validated, and applied to evaluate herbal and pharmaceutical antimalarial formulations (HPAFs) and starting materials (APIs). The separation/quantitation of the alkaloids (including quinine, quinidine, cinchonine, cinchonidine, dihydroquinine, dihydroquinidine, and cryptolepine) was achieved on a Zorbax SB-CN column $(250 \mathrm{~mm} \times 4.6 \mathrm{~mm}, 5 \mu \mathrm{m})$, with an isocratic elution system of methanol: trifluoroacetic acid $(0.1 \%$, v/v) $(15: 85$, v/v) at $1.5 \mathrm{~mL} / \mathrm{min}$ and $223 \mathrm{~nm}$. Method validation was according to ICH Q2(R1) guidelines. It was then used to assess the quality of APIs $(n=3)$ and HPAFs $(n=44)$ including quinine-based pharmaceutical antimalarial formulations (QBPAFs) $(n=23)$ and herbal antimalarial products (HAMPs). The method was found to be specific, selective, accurate, precise, and robust toward the alkaloids with linearity achieved within specified concentration ranges $\left(r^{2}>0.995\right.$ for all analytes). Analyte stability ranged between 6 and 12 hours. All the APIs contained quinine $<99.0 \%-101.0 \%$, with dihydroquinine and cinchonidine at levels compliant with the established acceptance criteria. The QBPAFs had quinine content ranging between $50.2 \%$ and $151.2 \%$, with $43.5 \%(n=10 / 23)$ of them complying with the acceptance criteria. The related alkaloids observed in the QBPAFs included quinidine $(56.5 \%, n=13 / 23)$, dihydroquinine ( $100 \%, n=23 / 23)$, dihydroquinidine $(21.7 \%, n=5 / 23)$, cinchonine $(17.4 \%, n=4 / 23)$, and cinchonidine (95.7\%, $n=22 / 23)$. For the HAMPs, $81.0 \%(n=17 / 21)$ were adulterated with quinine $(0.59 \pm 0.04 \mathrm{mg} / 10 \mathrm{~mL}-86.03 \pm 0.02 \mathrm{mg} / 10 \mathrm{~mL})$. Cryptolepine was identified in $19 \%(n=4 / 21)$ of the HAMPs with concentration ranging between $43.99 \pm 0.43 \mu \mathrm{g} / \mathrm{mL}$ and $747.86 \pm 0.34 \mu \mathrm{g} / \mathrm{mL}$. In conclusion, the application of the ion-pair HPLC method targeting quinoline and indoloquinoline antimalarials has demonstrated the presence of quality and poor-quality HPAFs on the Ghanaian market. 


\section{Introduction}

In recent years, significant progress has been made in the control of malaria on the African continent. Between 2000 and 2019 , the incidences of malaria declined by $29 \%$ whereas deaths declined by $60 \%$. That notwithstanding, a significant proportion of the African population continues to remain at risk to the disease. For instance, in the year 2019, out of the 229 million malaria cases and 409,000 malaria deaths reported globally, $94 \%$ of them were recorded in the African subregion. Also, in 2020, 384,000 preventable malaria deaths were recorded in the subregion [1].

Due to the continuous-ravaging effects of malaria on human lives, there is a dedicated focus on the production of herbal and pharmaceutical antimalarial formulations (HPAFs). Currently, an increased production trend of HPAFs is observed in Sub-Saharan Africa. However, the production of substandard products could ultimately erode the gains made toward the fight against malaria [2].

Artemisinin-based combination therapies (ACTs) remain the first-line treatment options for uncomplicated malaria in Ghana since their introduction [3]. The Cinchona alkaloid quinine has also been used for a very long time, and it continues to serve its purpose in the management of malaria. It is used for uncomplicated malaria in the first trimester of pregnancy and, in some cases, in the second and third trimesters. It is also used in severe malaria and in treatment failures associated with the ACTs [3]. The use of quinine remains a common practice in malaria-endemic regions especially among pediatrics $[4,5]$. Although its mechanism of action remains unknown, quinine is known to exert schizonticidal effect against intraerythrocytic parasites and gametocytocidal effect against Plasmodium vivax and Plasmodium malariae. Also, quinine possesses analgesic effect, which makes it beneficial in malaria treatment [4]. However, it is well known for its toxic effects due to either supradrug levels or the presence of its related chiral derivatives, including, dihydroquinine, quinidine, dihydroquinidine, cinchonine, and cinchonidine. These toxic effects are commonly referred to as cinchonism. Although some of the derivatives are known to also possess antimalarial activity, their narrow range of physiological activity exposes them in terms of their toxic effects [6]. In addition to their toxic effects, the quality status of quinine products is at times brought to question. Unlike the ACTs which see regular quality assessments in the country, with their reports readily available [7-9], assessments of quinine products are limited. Considering that they are also widely used, especially among pediatrics and pregnant women and in severe malarial cases, their routine quality assessments are required.

Herbal medicines (HMs) continue to enjoy significant patronage among Ghanaians. Recently, the prevalence of $\mathrm{HM}$ use is put around $76.5 \%$ [10]. In terms of antimalarial products, a study by Yeboah et al. showed that herbal antimalarial products (HAMPs) are preferred to pharmaceutical antimalarial formulations (PAFs) because they are perceived to be more effective, with minimal side effects, and less expensive [11]. As a result, many resort to HAMPs as either their first-line, second-line, or in combination with PAFs for malaria treatment [11]. This has led to the proliferation of HAMPs on the market. Cryptolepis sanguinolenta roots have been traditionally used for several years to treat malaria and form one of the frequently occurring constituent in these HAMPs [12-14]. Its main constituent, cryptolepine, an indoloquinoline, possesses in vitro and in vivo antiplasmodial effects $[15,16]$. One of the major challenges of the herbal medicine manufacturing industry is product quality assurance. Issues of product falsification, counterfeiting, adulteration, and substandard quality have been previously reported. For these reasons, it is also pertinent to monitor the quality of HAMPs.

Although compendial methods exist for the analysis of quinine-based products, their scopes are limited. For example, United States Pharmacopoeia's monograph on quinine sulfate includes specifications on quinine, dihydroquinine, cinchonidine, and quininone [17]. The European Pharmacopoeia on the other hand has specifications on quinine and its related substances, namely, quinidine, dihydroquinidine, and dihydroquinine [18]. Though cinchonine is equally a well-known related substance of quinine, its control is not considered in the existing compendial methods. Also, previous methods developed for cinchona alkaloids and cryptolepine had employed either C18 or C8 columns and were faced with poor resolution and peak shape challenges as a result of their interactions with the silanol groups [19]. Ion exchange-type chiral stationary phases have proven beneficial [20], but due to their cost and limited availability, there is a need to explore other suitable and relatively affordable alternatives.

In order to monitor the quality of HPAFs with respect to these alkaloidal contents, with relatively better and affordable chromatographic conditions, there was a need to develop a new method. This study thus sought to develop and validate an ion-pair HPLC method simultaneously targeting quinoline (quinine, quinidine, cinchonine, cinchonidine, dihydroquinine, and dihydroquinidine) and indoloquinoline (cryptolepine) alkaloids (Figure S1) in HPAFs on the Ghanaian market.

\section{Materials and Methods}

2.1. Study Design. The study was conducted in two phases. The first phase was on the development and validation of the ion-pair HPLC method in accordance with the International Conference on Harmonization (ICH Q2(R1)) guidelines. The second phase involved using the developed method in a cross-sectional study on the quality of HPAFs by adopting a purposive sampling approach to sample the products from the consumer market.

\subsection{Standards and Samples}

2.2.1. Reference Standards. Reference standards of quinine, quinidine, cinchonine, cinchonidine, dihydroquinine, and hydroquinidine were purchased from Glentham Life Sciences, United Kingdom. The cryptolepine reference standard was purchased from Sigma-Aldrich, USA. 
2.2.2. Samples. The HPAFs obtained for the study included APIs $(n=3)$ (Table S1), quinine-based pharmaceutical antimalarial formulations (QBPAFs) $(n=23)$ (Table S2), and HAMPs $(n=21)$ (Table S3). The QBPAFs and HAMPs were purchased from different outlets in Accra and Kumasi, while the APIs were donated from three different local pharmaceutical manufacturing companies. All samples were handled in accordance with the recommendations of respective manufacturers.

2.3. Instrumentation and Equipment. The HPLC system used was a 1260 series Agilent technology high-performance liquid chromatograph equipped with infinite variable wavelength detector (VWD), autosampler, vacuum degasser, thermostat column compartment, and 1260 series quaternary pump. The analysis and data integration were performed using Agilent OpenLab CDS ChemStation Edition for LC \& LC-MS Systems (Agilent Technologies; 2016).

\subsection{Preparation of Solutions}

2.4.1. Standard Solutions. Stock solutions of quinidine, cinchonidine, cinchonine, dihydroquinine, dihydroquinidine, and cryptolepine were prepared by dissolving $4 \mathrm{mg}$ each of the reference standards in $10 \mathrm{~mL}$ of mobile phase to obtain a concentration of $400 \mu \mathrm{g} / \mathrm{mL}$ each. These were diluted to the working concentration of $80 \mu \mathrm{g} / \mathrm{mL}$. For quinine sulfate, a stock concentration of $2,000 \mu \mathrm{g} / \mathrm{mL}$ was prepared by dissolving $20 \mathrm{mg}$ in $10 \mathrm{~mL}$ of the mobile phase and then diluted to a working concentration of $400 \mu \mathrm{g} / \mathrm{mL}$.

\subsubsection{Sample Solutions}

(1) Quinine Sulfate API Samples. An accurate weight of $50 \mathrm{mg}$ of each API sample $(n=3)$ was weighed and dissolved with the mobile phase in a $100 \mathrm{~mL}$ volumetric flask to produce a concentration of $500 \mu \mathrm{g} / \mathrm{mL}$. The sample solutions were then analyzed with the conditions described in Section 2.5 .

(2) Quinine-Based Pharmaceutical Antimalarial Formulations. For QBPAFs $(n=23)$, including syrups $(n=19)$, tablets $(n=1)$, and injectables $(n=3)$, an accurate amount of each product (volume or weight) containing an equivalent weight of $50 \mathrm{mg}$ quinine base was taken, transferred into $100 \mathrm{~mL}$ volumetric flask, dissolved with the mobile phase, and topped up with same to the required mark. Each sample solution was then sonicated for 15 minutes, diluted serially to produce $500 \mu \mathrm{g} / \mathrm{mL}$ concentration, and filtered with a Whatman No. 1 filter paper. The final sample solutions were then accordingly analyzed using the chromatographic conditions described in Section 2.5 .

(3) Herbal Antimalarial Products. For the herbal products $(n=21), 10 \mathrm{~mL}$ of each sample was pipetted and dissolved with mobile phase in a $100 \mathrm{~mL}$ volumetric flask with sonication for 15 minutes. The solutions were filtered with a Whatman No. 1 filter paper and then analyzed using the chromatographic conditions in Section 2.5.

\subsection{Method Development and Validation}

2.5.1. Chromatographic Conditions. The chromatographic separations were performed using Zorbax SB-CN column $(250 \mathrm{~mm} \times 4.6 \mathrm{~mm}, 5 \mu \mathrm{m})$ at a column temperature of $40^{\circ} \mathrm{C}$, with an isocratic elution system using a mobile phase of methanol: trifluoroacetic acid $(0.1 \%, \mathrm{v} / \mathrm{v})$ and $(15: 85, \mathrm{v} / \mathrm{v})$ at a flow rate of $1.5 \mathrm{~mL} / \mathrm{min}$. The injection volume was $2 \mu \mathrm{L}$, and detection was at a wavelength of $223 \mathrm{~nm}$.

2.5.2. Method Validation. The ion-pair HPLC method developed was then validated according to the ICH Q2(R1) guidelines with respect to specificity and selectivity, linearity, limit of detection (LOD), limit of quantification (LOQ), precision, accuracy, robustness, and stability of solutions [21].

(1) Specificity/Selectivity. This was demonstrated by comparing the chromatograms from the diluent (mobile phase system) with that from the working standards at a concentration level of $80 \mu \mathrm{g} / \mathrm{mL}$ for quinidine, cinchonine, cinchonidine, dihydroquinine, dihydroquinidine, and cryptolepine (100\% working) and $400 \mu \mathrm{g} / \mathrm{mL}$ for quinine sulfate [22].

(2) Linearity. Linearity was determined using concentration ranges $10-200 \mu \mathrm{g} / \mathrm{mL}$ for quinidine, cinchonine, cinchonidine, dihydroquinine, dihydroquinidine, and cryptolepine, and $50-1000 \mu \mathrm{g} / \mathrm{mL}$ for quinine sulfate. Linear regression models were calculated from triplicate determinations of peak areas with their respective concentrations. Linearity was evaluated from the correlation coefficient, the residuals, $y$-intercept, and slope [23].

(3) Limit of Detection and Limit of Quantification. The limits of detection (LOD) and limits of quantification (LOQ) were determined from the linear regression models as per the $\mathrm{ICH}$ Q2(R1) guidelines.

(4) Precision. Intraday and interday precisions were investigated for all analytes at concentration levels corresponding to $80 \%, 100 \%$, and $120 \%$ of respective working standards. For intraday precision, triplicate analysis of concentrations at the three levels was determined in the same day. For interday precision, similar investigations at the same concentration levels were carried out on different days. In both cases, precision was determined from the estimation of the relative standard deviation and ANOVA analysis $[21,23]$.

(5) Accuracy. Method accuracy was investigated by determining the recoveries of the analytes at $80 \%, 100 \%$, and $120 \%$ of respective working standard concentrations [21]. 
(6) Robustness. Concentrations of the analytes corresponding to $100 \%$ were analyzed using two different columns from different manufacturers. The percentage contents for the analytes were then statistically compared [21].

(7) Stability of Solution. The stability of the analytes' solutions was evaluated over 48 hours using concentrations of the analytes equivalent to $100 \%$ concentrations after preparation. The solutions were analyzed at following time intervals: $0,0.5,1,3,6,9,12,24,30,36$, and 48 hours. All determinations made were in triplicates, and the results were analyzed using regression analysis [23].

2.6. Analysis of Samples. The validated method was used to analyze the HPAFs and the APIs. The contents of the target analytes in the samples were assessed from triplicate peak area determinations by applying the obtained linear regression models.

2.7. Data Analysis. Data from the method validation were analyzed using descriptive statistics (including mean, standard deviation, and relative standard deviation), inferential statistics (included Student's $t$-test, and one- and twoway ANOVA), and regression analyses from GraphPad Prism for Windows (version 6.01, 2012). The quality of the QBPAFs was further explored using the unsupervised multivariate methods, principal component analysis (PCA), and hierarchical cluster analysis (HCA) from Minitab 18 (Minitab Inc., 2017). Cluster modeling was achieved using the complete linkage method with squared Euclidean distance. Prior to the analysis, the data were preprocessed by standardizing each column with their respective means and standard deviations.

\section{Results}

3.1. Method Development and Validation. UV/Vis scans of all the seven compounds of interest in methanol showed a common UV absorption maximum at $223 \mathrm{~nm}$, and this wavelength was selected for the detection. Due to the poor resolution associated with $\mathrm{C} 18$ and $\mathrm{C} 8$ columns [19], a CNbonded column was employed in this study and the results showed a good peak resolution and symmetry (Figure 1). The optimized mobile phase composition was methanol: trifluoroacetic acid $(0.1 \%, \mathrm{v} / \mathrm{v})(15: 85, \mathrm{v} / \mathrm{v})$. A flow rate of $1.5 \mathrm{~mL} / \mathrm{min}$ was observed to ensure the good resolution of the analytes, with mean retention times being significantly different from each other $\left(\mathrm{F}_{[6,14]}=262.6 ; p<0.0001\right)$ (Figure 1; Table 1).

The method was subsequently validated in accordance with the ICH Q2(R1) guidelines [21]. The method developed was able to identify each of the analytes without interference from the mobile phase and the sample matrix. The method was thus shown to be selective and specific toward all the compounds investigated. It was also shown to be precise in both intraday and interday investigations, linear within specified concentration ranges, accurate, and robust. The minimum stability of the analyte solutions was 6 hours after preparation. The outcome of the validation is summarized in Table 1 and Figure 2.

\subsection{Analysis of Samples}

3.2.1. Overview of Samples. A total of 47 antimalarial samples were collected from Kumasi $(63.8 \%, n=30 / 47)$ and Accra $(36.2 \%, n=17 / 47)$ for the analyses. This included herbal products $(44.7 \%, n=21 / 47)$, with some containing cryptolepine as per their label claim (33.3\%, $n=7 / 21)$, quinine-containing orthodox products $(48.9 \%, n=23 / 47)$, and quinine sulfate APIs $(6.4 \%, n=3 / 47)$. All the herbal products presented as syrups $(100 \%, n=21 / 21)$, while the orthodox products presented as syrups $(82.6 \%, n=19 / 23)$, tablets $(4.3 \%, n=1 / 23)$, and injections $(13.0 \%, n=3 / 23)$. The products were observed to have originated from four countries, including Ghana $(83.0 \%, n=39 / 47)$, India $(12.8 \%$, $n=6 / 47)$, Democratic Republic of Congo $(2.1 \%, n=1 / 47)$, and the United Kingdom $(2.1 \%, n=1 / 47)$. Table S4 shows a brief overview on the samples analyzed.

3.2.2. Quinine Starting Materials. The APIs analyzed contained concentrations of quinine less than the acceptable levels of $99.0 \%-101.0 \%$ (Figure 3(a)) [18]. The related substances, including dihydroquinine and cinchonidine, were detected. While cinchonidine levels ranged between $0.74 \pm 0.08 \%$ and $1.57 \pm 0.04 \%$, dihydroquinine ranged between $5.04 \pm 0.02 \%$ and $7.40 \pm 0.07 \%$. In each case, their levels were compliant with their respective acceptance criteria [18]. In the API category of samples, dihydroquinidine, cinchonine, and quinidine were either absent or below the detection limit.

\subsubsection{Quinine-Based Pharmaceutical Antimalarial} Formulations. All the QBPAFs contained quinine $(100 \%$, $n=23 / 23$ ) as per their label claims. Residues of related substances (present as impurities) were also found in some of them, and this included quinidine $(56.5 \%, n=13 / 23)$, dihydroquinine (100\%, $n=23 / 23)$, dihydroquinidine $(21.7 \%, n=5 / 23)$, cinchonine $(17.4 \%, n=4 / 23)$, and cinchonidine $(95.7 \%, n=22 / 23)$. In this category of samples, the quinine concentrations ranged between $50.2 \%$ and $151.2 \%$ (median $=97.34 \%$ ) (Figure 3(b)). There were $43.5 \%(n=10 / 23)$ of these products that complied with the acceptance criteria of $90 \%-110 \%$ for content assay [17]. Out of the $56.5 \%(n=13 / 23)$ that did not comply, $46.2 \%$ $(n=6 / 13)$ of them recorded contents above $110 \%$ and $53.8 \%$ $(n=7 / 13)$ had contents below $90 \%$. Furthermore, while all the injections were found to be compliant $(100 \%, n=3 / 3), 52.2 \%$ $(n=12 / 19)$ of the syrups and the only tablet formulation investigated were not complaint with the acceptance criteria (Figure 3(c)). In relation to the related substances, cinchonidine and dihydroquinine levels were observed to be below the maximum limit of $5 \%$ and $10 \%$, respectively [17].

The outcomes of the multivariate data analysis are summarized in Figures 4(a) and 4(b). From the PCA, the variability in the contents of the analytes was explained by 


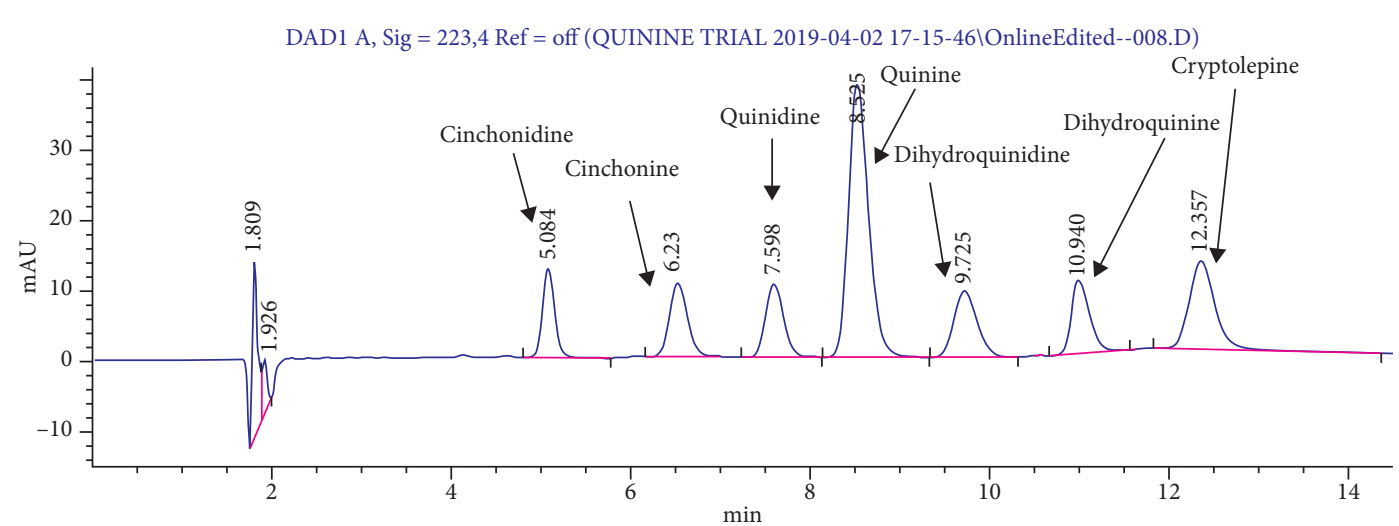

(a)

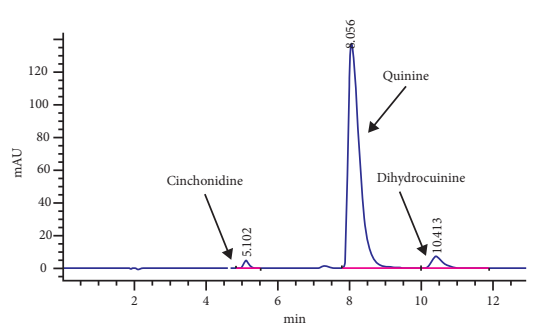

(b)

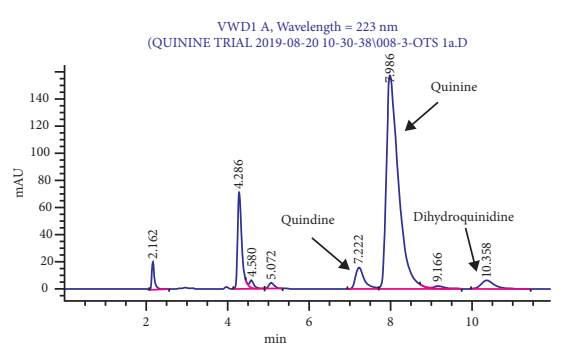

(c)

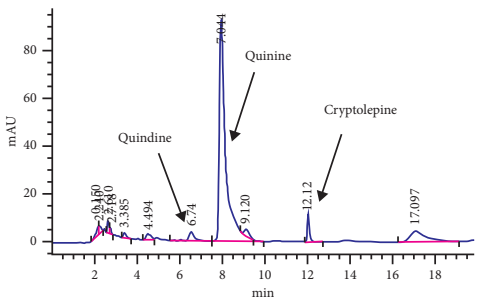

(d)

FIGURE 1: HPLC chromatograms of reference standards and samples using the developed method. (a) Chromatogram showing the optimum separation of the reference standards, namely, cinchonidine, cinchonine, quinidine, quinine, dihydroquinidine, dihydroquinine, and cryptolepine using the developed method. (b) Chromatogram showing the presence of quinine, cinchonidine, and dihydroquinine in an API sample. (c) Chromatogram showing the presence of quinine, quinidine, and dihydroquinidine in a QBPAF. (d) Chromatogram showing the presence of quinine, quinidine, and cryptolepine in a HAMP.

the first two components, PC1 (37.1\%) and PC2 (27.2\%). While PC1 partly explained the variability in the levels of quinine, quinidine, dihydroquinidine, cinchonine, and cinchonidine, PC2 partly explained the variability in the levels of dihydroquinine, cinchonine, dihydroquinidine, and quinidine. The score plot (Figure 4(a)) showed some level of clustering of some samples from their PC scores. Samples with similar PC scores are thought to exhibit similar characteristics in terms of the levels of the analytes monitored. It was thus observed that some samples from different batches from the same manufacturer exhibited similar PC scores, thereby showing up as clusters of scores. For example, products OTS7a-OTS7c originated from the same manufacturer and were seen to have similar scores. Similarly, OTS1a-OTS1d also originated from the same manufacturer. There were, however, other products from the same manufacturer that had different scores, indicating some sort of batch-to-batch differences. For example, OTS3a-OTS3e also originated from the same manufacturer, but scores for
OTS3e and OTS3a were very different. These observations were confirmed from HCA, where cluster amalgamation led to the observation of clusters consistent with that observed from the PC scores of the samples (Figure 4(b)).

Essentially, there were three main clusters observed and these are briefly described below: the first cluster, with a similarity of $53.89 \%$, comprised 6 syrup products: four different batches from a first local manufacturer and two batches from a second local manufacturer, who also had other batches having different quality attributes. These products were characterized by the presence of dihydroquinidine $(0.03 \pm 0.03 \%-0.15 \pm 0.01 \%, n=6 / 6)$, higher levels of quinidine $(2.18 \pm 0.03 \%-2.8 \pm 0.36 \%, n=6 / 6)$, and quinine contents, most of which were within the acceptance criteria $(60 \%, n=4 / 6)$. The four which were compliant originated from the first local manufacturer, while the two which were not compliant were from the second local manufacturer. The second cluster, with a similarity of $70.86 \%$, comprised of 4 syrup products, three of which were 
TABLE 1: Summary of validation results for the developed method.

\begin{tabular}{|c|c|c|c|c|c|c|c|}
\hline & Cinchonidine & Cinchonine & Quinidine & Quinine & Dihydroquinidine & Dihydroquinine & Cryptolepine \\
\hline Retention times & $5.10 \pm 0.14$ & $6.08 \pm 0.13$ & $7.25 \pm 0.11$ & $8.12 \pm 0.23$ & $9.35 \pm 0.30$ & $10.19 \pm 0.27$ & $12.32 \pm 0.48$ \\
\hline LDR $(\mu \mathrm{g} / \mathrm{mL})$ & $10-200$ & $10-200$ & 10-200 & $50-1000$ & $10-200$ & 10-200 & 10-200 \\
\hline$r^{2}$ & 0.9995 & 0.9976 & 0.9996 & 0.9985 & 0.9990 & 0.9994 & 0.9984 \\
\hline $\mathrm{LOD}(\mu \mathrm{g} / \mathrm{mL})$ & 3.83 & 8.18 & 3.30 & 32.01 & 5.12 & 3.99 & 6.74 \\
\hline LOQ $(\mu \mathrm{g} / \mathrm{mL})$ & 11.61 & 24.79 & 10.00 & 97.00 & 15.52 & 12.11 & 20.42 \\
\hline $\begin{array}{l}\text { Intraday precision } \\
(\% \text { RSD })\end{array}$ & 1.13 & 0.89 & 0.89 & 0.79 & 0.90 & 1.13 & 0.45 \\
\hline $\begin{array}{l}\text { Interday precision } \\
\text { (\% RSD) }\end{array}$ & 0.76 & 0.68 & 0.73 & 0.79 & 0.67 & 0.83 & 0.55 \\
\hline $\begin{array}{l}\text { Accuracy } \pm \text { SD } \\
\text { (\% recovery) }\end{array}$ & $99.53 \pm 0.7103$ & $99.73 \pm 0.8459$ & $100.1 \pm 0.9295$ & $100.2 \pm 1.157$ & $99.93 \pm 1.1016$ & $99.50 \pm 0.9031$ & $99.69 \pm 0.6641$ \\
\hline
\end{tabular}

(\% recovery)

Robustness

Stability of analyte

No significant differences in the contents of analytes from the two columns solutions (hrs.) 6 6 9 9 6 6 12

Linear dynamic range (LDR), coefficient of correlation $\left(r^{2}\right)$, LOD, and LOQ were determined from linear regression curves.

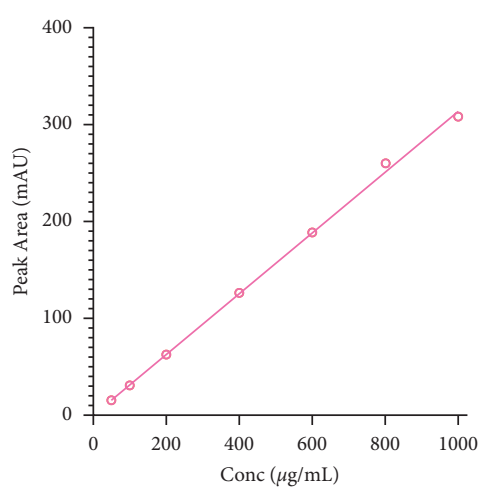

$\multimap$ QUIN

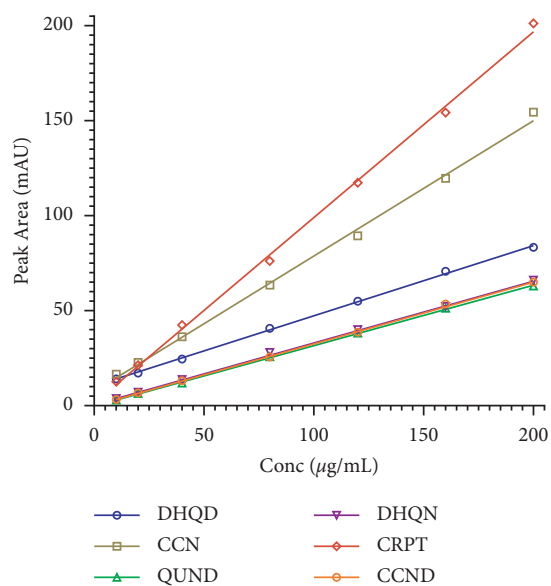

(b)

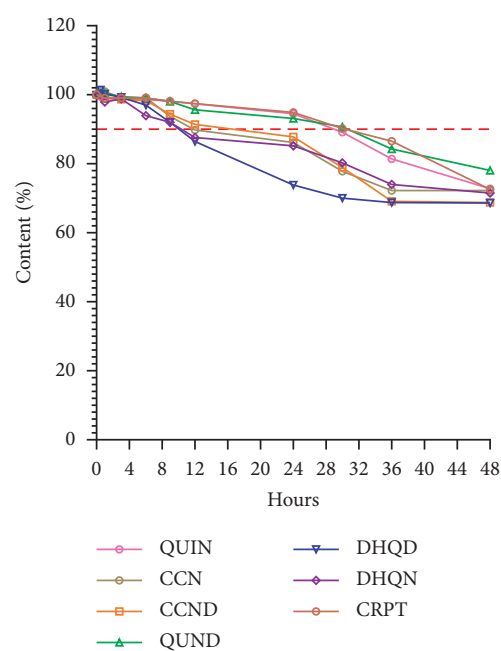

(c)

FIGURE 2: Results for linearity and stability studies in the method validation. (a) Linearity responses for quinine over the concentration range $50-1000 \mu \mathrm{g} / \mathrm{mL}$. (b) Linearity responses for all other analytes each at concentration range $10-200 \mu \mathrm{g} / \mathrm{mL}$. (c) Stability of analyte solution studies. The red horizontal line indicates the lowest permissible content of analytes ( $90 \%$ of starting concentrations). The stability of the analyte solutions ranged between 6 and 12 hours after sample preparation.

different batches from a third local manufacturer and the fourth was a product from India. These products were characterized by the presence of cinchonine $(0.67 \pm 0.01 \%-$ $1.86 \pm 0.01 \%)(100 \%, n=4 / 4)$ and higher quinine contents $(>100 \%)$, most of which were above $110 \%(75 \%, n=3 / 4)$. The third cluster, with a similarity of $56.32 \%$, comprised of 13 products, including tablets $(n=1)$, injections $(n=3)$, and syrups $(n=9)$. Some of these products complied with the acceptance criteria for quinine $(38.5 \%, n=5 / 13)$ while the others did not $(61.5 \%, n=8 / 13)$. These products were also characterized by the absence of cinchonine and dihydroquinidine as impurities in them.

3.2.4. Herbal Products. It was observed that $81.0 \%$ of the HAMPs $(n=17 / 21)$ contained varied concentrations of quinine, while $19.0 \%$ contained cryptolepine $(n=4 / 21)$. One product contained both quinine and cryptolepine at levels of
$66.75 \pm 0.13 \mathrm{mg} / 10 \mathrm{~mL}$ and $55.27 \pm 0.91 \mu \mathrm{g} / \mathrm{mL}$, respectively. The quinine concentrations detected ranged between $0.59 \pm 0.04$ and $86.03 \pm 0.02 \mathrm{mg} / 10 \mathrm{~mL}$ (median $=26.47 \mathrm{mg} / 10 \mathrm{~mL}$ ) and that of cryptolepine ranged between $43.99 \pm 0.43$ and $747.86 \pm 0.34 \mu \mathrm{g} / \mathrm{mL}($ median $=59.19 \mu \mathrm{g} / \mathrm{mL})($ Figure $3(\mathrm{~d})$ ).

\section{Discussion}

4.1. Method Development. The new method was developed as a result of the limitations with respect to the scope of target analytes associated with existing compendial and other published analytical methods for Cinchona alkaloids [17-19]. As indicated above, the compendial methods for quinine sulfate included specifications for a relatively limited number of related substances in addition to quinine. It was also noted that existing methods do not simultaneously analyze the contents of quinine, its related substances, and cryptolepine. As these are thought to be the main constituents in the non-ACTs used for 


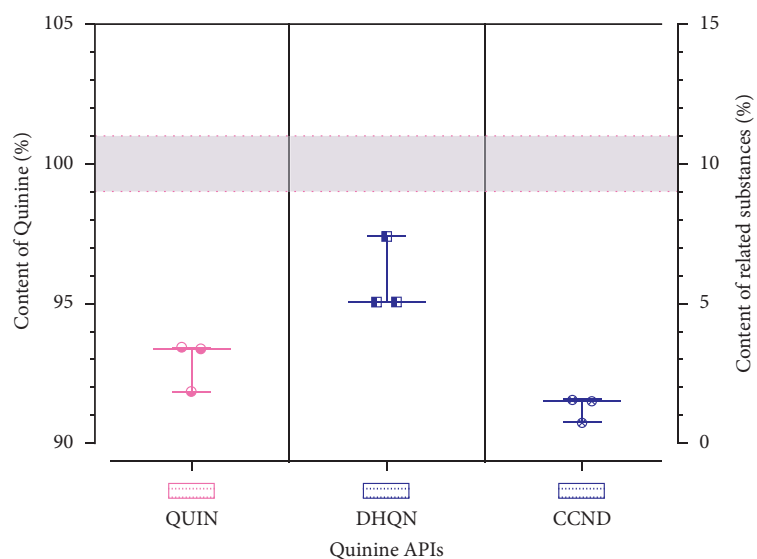

(a)

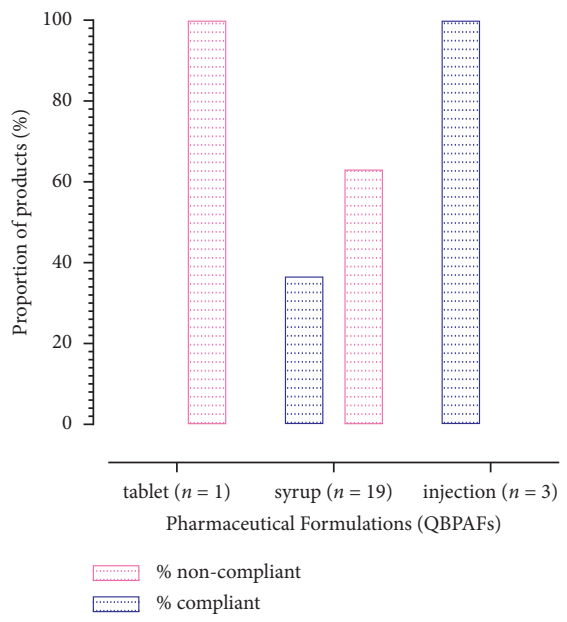

(c)

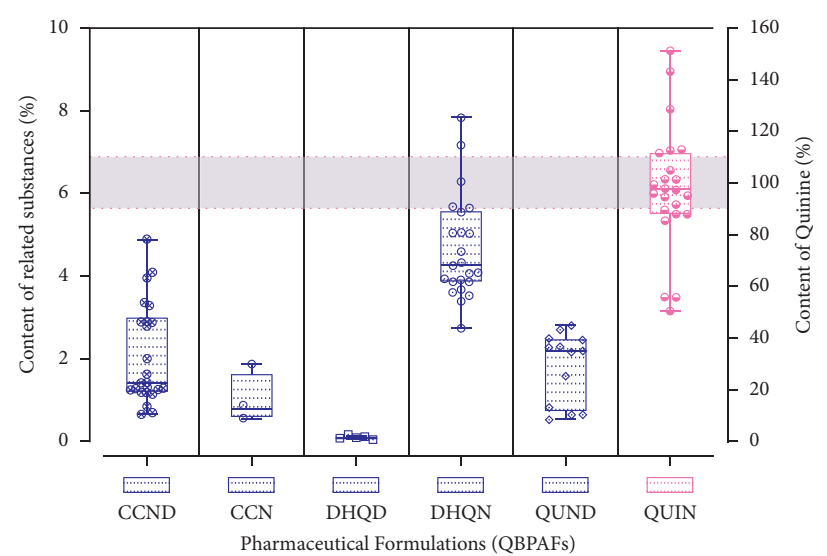

(b)

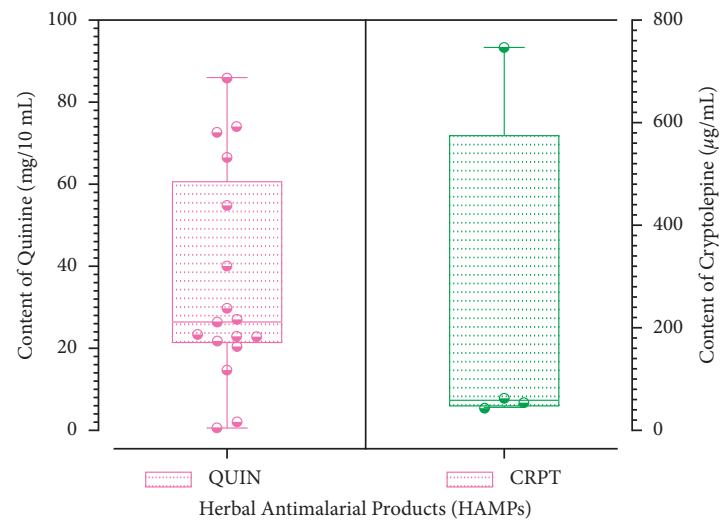

(d)

FIGURE 3: Analyses of contents in the herbal and pharmaceutical antimalarial formulations. (a) Content assays of quinine APIs $(n=3)$. Quinine contents (Ref: left $y$-axis) and related substances (Ref: right $y$-axis) detected. None of the APIs contained the right amount of quinine (indicated as the highlighted region in the graph) for product manufacture. (b) Contents of quinine (Ref: right $y$-axis) and its related alkaloidal substances (Ref: left $y$-axis) in the orthodox products. The highlighted region (Ref: right $y$-axis) indicates the range of contents deemed acceptable for quinine content. (c) Proportions of QBPAFs that either complied or did not comply with the acceptance criteria on quinine content. (d) Content assays of analytes in the herbal products. Quinine (Ref: left $y$-axis) and cryptolepine (Ref: right $y$-axis) constitute the main antimalarial alkaloids detected in the herbal products. Quinine (QUIN) and cryptolepine (CRPT) constitute the main APIs in the samples analyzed, while the related substances included cinchonidine (CCND), cinchonine (CCN), dihydroquinidine (DHQD), dihydroquinine (DHQN), and quinidine (QUND).

malarial therapy in Ghana, any new method being developed would be more beneficial if it targeted both quinoline (Cinchona) and indoloquinoline (cryptolepine) alkaloids. Hence, the need for this new method developed.

The chemistry of the alkaloids, including solubility, $\mathrm{pKa}$, absorptivity, and polarity, was considered in selecting optimum mobile phase, column, wavelength of absorption, temperature, and mode of elution for the method developed. The stable bond (SB) cyano column was chosen due to its exceptional stability at low $\mathrm{pH}$ and ability to withstand high temperature up to $90^{\circ} \mathrm{C}$, and its use ensures good peak resolution of basic compounds [24]. Such columns also possess an intermediate polarity, which makes them suitable in both reverse- and normal-phase HPLC methods. The outcome of the validation showed that the developed method was suitable for analysis and the quality control of the contents of the quinoline and indoloquinoline alkaloids.
4.2. Adulterated and Substandard Herbal Antimalarials. Due to the acceptability and wide patronage of herbal medicines, and high perception of their safety and efficacy because of their natural origins [11], coupled with systemic challenges in their proper regulation in Ghana, the herbal industry has become vulnerable and a fertile ground for exploitation by unscrupulous persons who only aim to maximize profit with no regard to human safety [25]. One key resultant effect of such systemic lapses is the issue of adulteration. Some manufacturers resort to adding known pharmaceuticals to their herbal products in the quest to enhance product efficacy [26]. Previous studies in the country have shown the presence of adulterants like dexamethasone [27] and sulfur [28] in some topical herbal products. Elsewhere, a number of reports on adulteration involving herbal medicines have been documented [29]. 


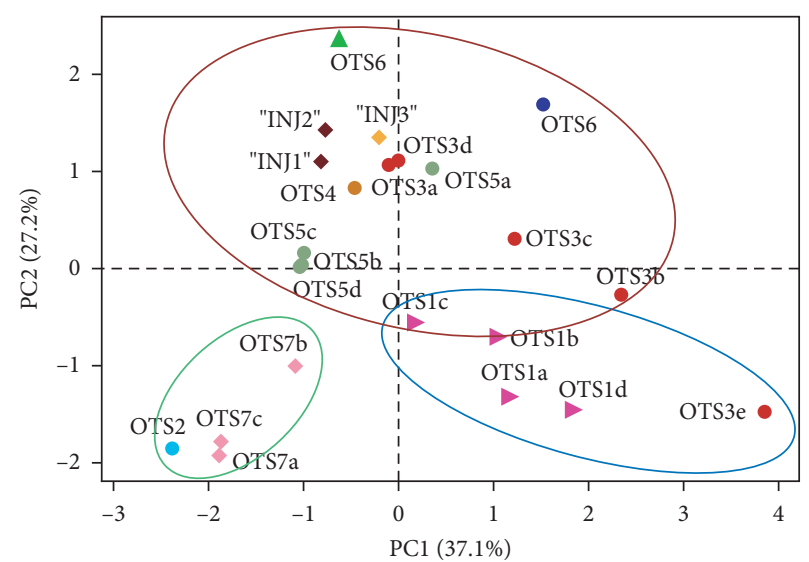

(a)

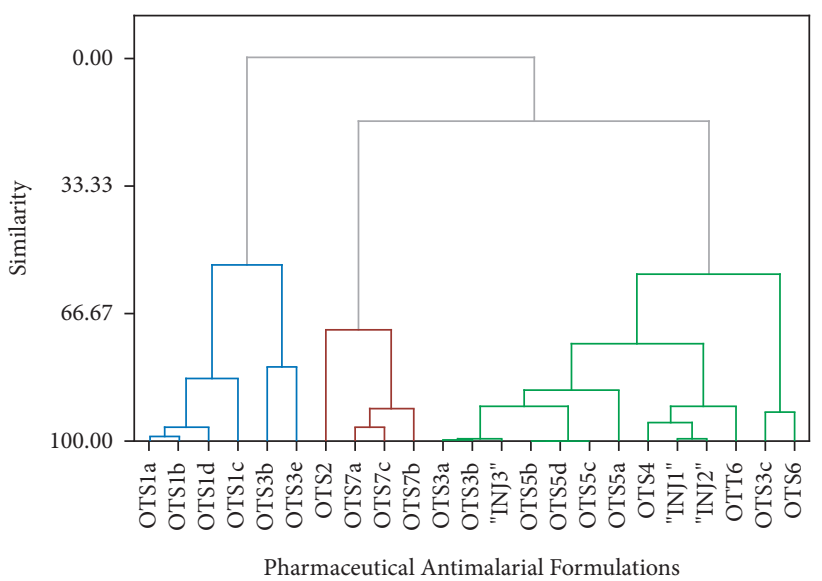

(b)

Figure 4: Multivariate data analysis of analytes contents in the quinine-based pharmaceutical antimalarial formulations. (a) Score plot from the principal component analysis showing some similarities and dissimilarities among the investigated products. Products with the same color symbols originate from the same manufacturer. (b) Dendrogram from hierarchical cluster analysis showing the clustering of the samples as determined from the contents of quinine and its related alkaloidal substances in the products.

Authors in this study report the likely incidence of adulteration of the HAMPs with quinine. It is worthy to note that none of the HAMPs sampled had a label claim of Cinchona sp. as a plant constituent or Cinchona alkaloids as labeled constituents. Recently, it has been reported that the main plant constituents in HAMPs, as documented in the Recommended Essential Herbal Medicine List (REHML) in Ghana, include Cryptolepis sanguinolenta (Lindl.) Schltr, Azadirachta indica A. Juss., and Alstonia boonei De Wild [14]. Previous ethnobotanical and ethnopharmacological studies conducted in different parts of the country confirm the presence of the above-mentioned plants, together with others for the treatment of malaria [12, 30,31]. In none of such surveys was there a report of the use of Cinchona sp. by traditional folks for their treatment or its presence as one of the plant constituents in HAMPs [12, 14, 30, 31]. The apparent detection of quinine in significant quantities in the majority of the sampled HAMPs may therefore indicate the possibility of deliberate adulteration by the respective manufacturers with the compound to enhance product efficacy. From the wide range of the quinine concentrations detected in these products (Figure 3(d)), it is obvious that the levels of quinine added are not standardized and properly controlled, and this could pose dangerous health risks to consumers who patronize them [32]. There is also the risk of resistance development by Plasmodium parasites from exposures to subtherapeutic doses of quinine in some of these products.

In the case of cryptolepine, the compound was only observed in 4 out of the 7 products, which claimed Cryptolepis sanguinolenta as one of their plant constituents. This observation was similarly reported by Mensah et al. [33], and it was argued that this could be due to either wrong labeling of the herbal products with the plant or poor harvesting practices, which tend to affect the concentration of the compound [26]. In either of the proposed situations, there arise quality control and assurance concerns since the affected products may not be therapeutically effective and consumers would also not have value for money from purchasing them.

\subsection{Substandard Pharmaceutical Antimalarial Formulations.} Contrary to HAMPs, PAFs have a well-structured regulatory framework in Ghana that ensures that manufacturers strictly adhere to good manufacturing practices. That notwithstanding, there continue to be records of substandard antimalarials over the years and in several of these instances, the FDA had cause to issue product recalls from the market [34]. From the findings in this study, authors also report likely incidences of substandard QBPAFs from among the selected for the study.

The very low- and high-percentage contents of quinine in the noncompliant products could partly be due to the use of poor-quality APIs. The three APIs donated by the three independent manufacturers analyzed in this study were all found to be substandard (assay $<99.0 \%$ ). If manufacturers use API materials of such quality for their formulations, their products will likely end up with quinine contents lower than expected. In addition, if these substandard APIs were used and their quantities duly adjusted in formulations to compensate for their lower quinine levels (based on in-house analysis), accumulation of related substances (or impurities) may occur, and this will also be considered as undesirable. We argue that this could also be a reason for the observation in this study that the number of related substances in the APIs detected (that is, dihydroquinidine and cinchonidine) was less than that in the QBPAFs (that is, quinidine, dihydroquinine, dihydroquinidine, cinchonine, and cinchonidine). It may be possible that in the APIs, the related substances were present at levels that were below detection limits, but during manufacture, weight adjustments to compensate for substandard quinine levels could result in relatively higher weight being taken with higher amounts of 
these substances being detected and quantified. Also, if such adjustments are also not properly controlled, the products may rather end up with very high contents as also was evident for some of the products in this study.

Formulation challenges could have also contributed to the high incidence of substandard products. For instance, some of the noncompliant syrups were observed to have crystals in them (data not reported), suggesting a possible poor solubility of quinine sulfate and hence the low percentage contents of such products. Quinine is known to be poorly soluble in aqueous media, and its formulations have been widely investigated in attempts to improve upon solubility and bioavailability [35].

4.4. Product Quality Inconsistency. Every medicine consumer expects that his/her medicine is of the utmost reproducible quality from one batch to the other so that therapeutic efficacy for intended indications is guaranteed in the recommended time frame for treatment. It is therefore the responsibility of the manufacturer to institute robust quality assurance systems to guarantee such expectations. In this study, we identify instances of batch-tobatch variation in the quality attributes of the medicines investigated. From the PCA and HCA, it was observed that the QBPAFs were classified into three major groups depending on the similarities in their profiles with respect to quinine content and the contents of the related cinchona alkaloidal substances. In one of such instances, it was observed that samples OTS3a-OTS3e $(n=5)$ (Figure 4(a)), which originated from the same manufacturer, were classified in two different clusters. In one cluster, OTS3b and OTS3e had very low quinine contents of $55.73 \pm 0.10 \%$ and $50.23 \pm 1.01 \%$, respectively. They also contained dihydroquinidine, which was absent in the other three samples. In the second cluster, while OTS3c and OTS3d contained appreciable levels of quinine (that is, $95.01 \pm 0.04 \%$ and $91.64 \pm 1.54 \%$, respectively), OTS3a was found to be substandard with $89.51 \pm 0.21 \%$. Additionally, OTS3b, OTS3c, and OTS3e were also found to contain quinidine, and this was absent in OTS3a and OTS3d. These observations raise concerns about the quality of the quinine APIs used in manufacturing. Although the preliminary data gathered on these products show that they were manufactured at different times $(2016,2017,2018$, and 2019), for which reason it is possible to have used differently procured starting materials, it is worthy to note that for two of the samples produced in 2019, OTS3c and OTS3d, one contained quinidine and the other did not. This calls for a closer look at the quality of APIs used for product manufacturing in the country.

There was also an observation of three samples originating from one manufacturer, and all these samples recorded very high quinine contents (128.41 $\pm 1.07 \%$ $151.23 \pm 0.05 \%$ ), with the presence of quinidine, cinchonine, cinchonidine, and dihydroquinine. Obviously, it could be argued that such a manufacturer had challenges with the quality of the starting materials and challenges with the manufacturing process.
4.5. Risk of Toxicity from Accumulated Levels of Quinine and Its Related Substances. Substandard products appear either as products with very low or very high contents of the active ingredients or present with higher levels of impurities, and these are known to pose a dangerous health risk to medicine consumers. In the case of quinine, adverse effects like cinchonism, characterized by tinnitus, high-tone deafness, visual disturbances, headache, dysphoria, vomiting, and postural hypotension have been reported even with therapeutic doses [36]. The observation of supralevels of quinine in products indicated for use among children as evident from the current study is the more worrying. One study reported the death of one child with the other two presenting with major deficits from quinine poisoning [37]. High quinine levels have also been reportedly associated with retinal toxicity, with some leading to complete blindness, alteration of color vision, and blurriness [38, 39]. Additionally, the increasing levels of quinidine detected in a number of the samples also pose cardiotoxic risks such as postural hypotension, syncope, and QT interval prolongation, which may lead to arrhythmias [6].

\section{Conclusions}

This study reports on the development and validation of an ion-pair HPLC-UV method to monitor the quality of HPAFs containing quinoline and indoloquinoline alkaloids. Its application shows the prevalence of substandard quininebased pharmaceutical and cryptolepine-containing herbal antimalarials, and herbal antimalarials adulterated with quinine. In addition, APIs used to manufacture some of these products were observed to be substandard. In attempts to build on the successes achieved so far in the country toward the fight against malaria, as a key contributor to the general health and well-being of the Ghanaian populace, this study brings up topical quality assurance-related issues that threaten these successes and hence calls for considerations by regulatory agencies in the medicine quality landscape to intensify surveillance and introduce more training to curb the rising menace.

\section{Data Availability}

The processed data presented in this study and the information on the products sampled are included within this article and the Supplementary Information. All other information not included could, however, be obtained on request from the corresponding authors.

\section{Conflicts of Interest}

The authors declare that there are no conflicts of interest regarding the publication of this paper.

\section{Acknowledgments}

The authors are grateful to the three local manufacturing companies who donated samples of quinine APIs for the study. 


\section{Supplementary Materials}

The following supplementary information is attached: Tables S1, S2, S3, and S4; Figure S1. (Supplementary Materials)

\section{References}

[1] WHO, Message from the WHO Regional Director for Africa, WHO, Geneva, Switzerland, 2021.

[2] E. J. Walker, G. M. Peterson, J. Grech, E. Paragalli, and J. Thomas, "Are we doing enough to prevent poor-quality antimalarial medicines in the developing world?" BMC Public Health, vol. 18, no. 1, pp. 1-6, 2018.

[3] Ministry of Health, Anti-Malarial Drug Policy for Ghana, National Malaria Control Programme, Accra, Ghana, 2009.

[4] J. Achan, A. O. Talisuna, A. Erhart et al., "Quinine, an old antimalarial drug in a modern world: role in the treatment of malaria," Malaria Journal, vol. 10, pp. 1-12, 2011.

[5] B. Yir-Erong, M. T. Bayor, I. Ayensu, S. Y. Gbedema, and J. Boateng, "The challenges and knowledge gaps in malaria therapy: a stakeholder approach to improving oral quinine use in the treatment of childhood malaria in Ghana," Journal of Pharmaceutics, vol. 2018, pp. 1-12, 2018.

[6] A. Bykowski and T. D. Logan, "Cinchonism," in StatPearls, StatPearls Publishing, Treasure Island, FL, USA, 2021.

[7] M. El-Duah and K. Ofori-Kwakye, "Substandard artemisininbased antimalarial medicines in licensed retail pharmaceutical outlets in Ghana," Journal of Vector Borne Diseases, vol. 49, no. 3, pp. 131-139, 2012.

[8] D. Osei-Safo, A. Agbonon, D. Y. Konadu et al., "Evaluation of the quality of artemisinin-based antimalarial medicines distributed in Ghana and Togo," Malaria Research and Treatment, vol. 2014, Article ID 806416, 12 pages, 2014.

[9] M. Tivura, I. Asante, A. van Wyk et al., "Quality of Artemisinin-based combination therapy for malaria found in Ghanaian markets and public health implications of their use," BMC Pharmacology Toxicology, vol. 17, no. 1, p. 48, 2016.

[10] I. A. Kretchy, A. Koduah, K. F. M. Opuni et al., "Prevalence, patterns and beliefs about the use of herbal medicinal products in Ghana: a multi-centre community-based crosssectional study," Tropical Medicine \& International Health, vol. 26, no. 4, pp. 410-420, 2021.

[11] P. Yeboah, A. D. Forkuo, O. K. O. Amponsah et al., "Antimalarial drugs in Ghana: a case study on personal preferences," Science, vol. 2, no. 2, p. 28, 2020.

[12] G. Komlaga, C. Agyare, R. A. Dickson et al., "Medicinal plants and finished marketed herbal products used in the Treatment of malaria in the Ashanti region, Ghana," Journal of Ethnopharmacology, vol. 172, pp. 333-346, 2015.

[13] S. Osei-Djarbeng, E. Agyekum-Attobra, R. Nkansah, D. Solaga, S. Osei-Asante, and G. Owusu-Dapaah, "Medicinal plants constituting antimalarial herbal preparations in the Ghanaian market," British Journal of Pharmaceutical Research, vol. 5, no. 3, pp. 153-162, 2015.

[14] A. O. Agyemang, B. K. Turkson, M. F. Baidoo et al., "Utilization of plants for medicinal purposes and concerns with endangered plant species from Ghana," Traditional, Complementary and Integrative Medicine, vol. 6, no. 1, 2021.

[15] A. D. Forkuo, C. Ansah, K. M. Boadu et al., "Synergistic antimalarial action of cryptolepine and artemisinins," Malaria Journal, vol. 15, no. 1, p. 89, 2016.

[16] N. Osafo, K. B. Mensah, and O. K. Yeboah, "Phytochemical and pharmacological review of cryptolepis sanguinolenta (lindl.) schlechter," Advances in Pharmacological Sciences, vol. 2017, Article ID 3026370, 13 pages, 2017.

[17] USPC, Quinine Sulfate Capsules. In: United States Pharmacopoeia 32-National Formulary 27, United States Pharmacopeial Convention, Rockville, MD, USA, 2009.

[18] European Pharmacopoeia Commission, "Quinine sulphate," in European Pharmacopoeia, Council of Europe, Strasbourg, France, 2004.

[19] D. V. McCalley, "Analysis of the Cinchona alkaloids by highperformance liquid chromatography and other separation techniques," Journal of Chromatography A, vol. 967, no. 1, pp. 1-19, 2002.

[20] J. Teixeira, M. E. Tiritan, M. M. M. Pinto, and C. Fernandes, "Chiral stationary phases for liquid chromatography: recent developments," Molecules, vol. 24, no. 865, 2019.

[21] ICH, "Validation of analytical procedures: text and methodology Q2 (R1)," ICH Harmonised Tripartite Guideline, pp. 1-13, 2005.

[22] P. E. Goku, E. Orman, A. Naa, K. Quartey, J. K. Adu, and R. K. Adosraku, "A simple RP-HPLC method to simultaneously assay the contents of lamivudine, tenofovir, and nevirapine in fixed dose combined oral antiviral medicines," Journal of Chemistry, vol. 2020, Article ID 4618360, 9 pages, 2020.

[23] R. Bonfilio, E. C. L. Cazedey, M. B. d. Araújo, and H. R. Nunes Salgado, "Analytical validation of quantitative high-performance liquid chromatographic methods in pharmaceutical analysis: a practical approach," Critical Reviews in Analytical Chemistry, vol. 42, no. 1, pp. 87-100, 2012.

[24] Agilent Technologies, "ZORBAX $80 \AA$ StableBond," in The Essential Chromatography and Spectroscopy Catalog, pp. 264-266, Agilent Technologies, Santa Clara, CA, USA, 2012.

[25] M. Ekor, "The growing use of herbal medicines: issues relating to adverse reactions and challenges in monitoring safety," Frontiers in Pharmacology, vol. 4, p. 177, 2014.

[26] J. Zhang, B. Wider, H. Shang, X. Li, and E. Ernst, "Quality of herbal medicines: challenges and solutions," Complementary Therapies in Medicine, vol. 20, no. 1-2, pp. 100-106, 2012.

[27] K. Ofori-Kwakye, I. Ayensu, B. Akyinah, S. L. Kipo, and M. El Boakye-Gyasi, "Adulteration of Ghanaian topical herbal preparations with Dexamethasone," World Journal of Pharmacy and Pharmaceutical Sciences, vol. 3, no. 6, pp. 134-141, 2014.

[28] J. Addotey and M. M. S. Nyansah, "Quality assessment of some topical polyherbal preparations on the Ghanaian market," World Journal of Pharmacy and Pharmaceutical Sciences, vol. 5, no. 4, pp. 461-472, 2016.

[29] E. Ernst, "Adulteration of Chinese herbal medicines with synthetic drugs: a systematic review," Journal of Internal Medicine, vol. 252, no. 2, pp. 107-113, 2002.

[30] A. Adeniyi, A. Asase, P. K. Ekpe, B. K. Asitoakor, A. AduGyamfi, and P. Y. Avekor, "Ethnobotanical study of medicinal plants from Ghana; confirmation of ethnobotanical uses, and review of biological and toxicological studies on medicinal plants used in Apra Hills Sacred Grove," Journal of Herbal Medicine, vol. 14, pp. 76-87, 2018.

[31] K. S. Appiah, C. P. Oppong, H. K. Mardani et al., "Medicinal plants used in the ejisu-juaben municipality, southern Ghana: an ethnobotanical study," Medicines, vol. 6, no. 1, p. 1, 2018.

[32] T. H. Pearson and H.-P. Mannvik, "Quinine toxicity," Hydrobiologia, vol. 375-376, no. 4, pp. 317-329, 1998.

[33] A. Mensah, N. Kuntworbe, R. Johnson, and K. Ofori-kwakye, "Qualitative and quantitative assessment of cryptolepis-based 
herbal formulations within the Accra and Kumasi metropolis in Ghana," Journal of Innovations in Pharmaceutical and Biological Sciences, vol. 4, no. 1, pp. 58-65, 2017.

[34] FDA, Products Recall and Alert, Food and Drugs Authority, Accra, Ghana, 2021.

[35] S. Strauch, J. B. Dressman, V. P. Shah, S. Kopp, J. E. Polli, and D. M. Barends, "Biowaiver monographs for immediate-release solid oral dosage forms: quinine sulfate," Journal of Pharmaceutical Sciences, vol. 101, no. 7, pp. 2271-2280, 2012.

[36] J. S. McCarthy and R. N. Price, "Antimalarial drugs," in Mandell, Douglas, and Bennett's Principles and Practice of Infectious Diseases-Volume 1, J. E. Bennett, R. Dolin, and M. J. Blaser, Eds., pp. 495-509, Elsevier Inc, Amsterdam, Netherlands, 8th edition, 2015.

[37] T. M. Grattan-Smith, J. Gillis, and H. Kilham, "Quinine poisoning in children," Medical Journal of Australia, vol. 147, no. 2, pp. 93-95, 1987.

[38] H. N. Hall and A. J. Tatham, "Recovery from blindness following accidental quinine overdose," Practical Neurology, vol. 17, no. 6, pp. 469-471, 2017.

[39] J. Christoforidis, R. Ricketts, T. Loizos, and S. Chang, "Optical coherence tomography findings of quinine poisoning," Clinical Ophthalmology, vol. 5, no. 1, pp. 75-80, 2011. 